\title{
Flat and Conic Patches for Acute Ventricular Septal Perforation
}

\author{
Kanji Matsuzaki, Akihiko Ikeda, Taisuke Konishi, Tomoaki Jikuya
}

\begin{abstract}
Three surgical cases of acute ventricular septal perforation (VSP) successfully repaired with flat and conic patches are reported. The first case was a 58-year-old man who had undergone a double patch closure with two flat patches for VSP following acute myocardial infarction. On the third postoperative day, however, a redo operation became necessary due to recurrent VSP, and in this surgery flat and conic patches were used. The second and third cases were 82-year-old and 81-year-old women, respectively, who from the first underwent VSP closures with flat and conic patches. Although in the third case the patient died of hypoxic ischemic encephalopathy 2 months after operation, the others recovered well and were discharged at 2 months. Our procedure consists of an infarct exclusion technique and a double patch technique. The flat patch is for directly covering the infracted ventricular septum including the VSP through a left ventriculotomy. The conic patch is for three-dimensionally lining the infracted endocardium of the left ventricle. Similar procedures applying the same concept have been reported as effective for repairing acute VSP. The combination of both an infarct exclusion technique and a double patch technique can be thought of as an ideal strategy and our procedure provides one such option.
\end{abstract}

KEY WORDS: ventricular septal perforation, infarct exclusion technique, double patch technique

\section{Introduction}

Ventricular septal perforation (VSP) following acute myocardial infarction (AMI) is a rare but fatal complication. Though various techniques for repairing acute VSP have been introduced, its surgical mortality rate is still high. The Japanese Association for Thoracic Surgery has reported that its hospital mortality after operation was $38.3 \%$ in Japan during 2010. ${ }^{1)}$ Two important innovations have been made in regard to this type of surgery: an infarct exclusion technique and a double patch technique. Some surgeons have tried to integrate both these techniques, and we have done so using flat and conic patches. The details of our procedure as performed on three patients are reported here.

\section{Cases}

The first case was that of a 58-year-old man with chest pain and dyspnea, who was admitted to the emergency room. Echocardiography demonstrated a significant VSP shunt at the anterior ventricular septum, and coronary angiography showed 99\% stenosis of the left anterior descending artery (LAD), with a diagnosis of AMI complicated by a VSP. An intra-aortic balloon pump (IABP) was applied and an emergent surgery, consisting

Department of Cardiovascular Surgery, Tsukuba Medical Center Hospital, 1-3-1 Amakubo, Tsukuba, Ibaraki 305-8558, Japan

The essence of this paper was presented at the 26th Annual Meeting of the Japanese Coronary Association, Tokyo, December 15, 2012

Received August 5, 2013; Accepted November 17, 2013

doi: 10.7793/jcoron.19.13-00024 of VSP closure and coronary artery bypass grafting, was performed. In this surgery, the infarcted ventricular septum was repaired with two flat patches of bovine pericardium. The patient recovered well and was weaned off the IABP on the second postoperative day. On the following day, however, a redo operation became necessary due to recurrent VSP, in which we adopted a new procedure using flat and conic patches.

The second case was that of an 82-year-old woman who had suffered from AMI 1 week earlier and was admitted to our hospital because of deteriorating heart failure. Echocardiography demonstrated significant shunt flow through a VSP at the anterior ventricular septum, and coronary angiography showed a total occlusion of the LAD. The pulmonary-to-systolic flow ratio was 2.8 , so an IABP was applied and an emergent surgery was performed. For this patient, it was the intention from the very start to perform a VSP closure with flat and conic patches.

The third case was that of an 81-year-old woman with acute heart failure due to anteroseptal AMI with a large VSP, who was transferred to our emergency room. Echocardiography demonstrated large shunt flow through a VSP at the anterior ventricular septum. An IABP was applied in the emergency room and emergent surgery was performed as quickly as possible. Before cardiopulmonary bypass (CPB) was established, however, cardiopulmonary resuscitation was necessary because of cardiac arrest in the operating room. Fortunately, the patient recovered under CPB and a VSP closure was carried out. For this patient, flat and conic patches were used from the first.

In these cases, the times between the onset of AMI and surgery 
were 3 days, 1 week, and 1 day, respectively. We performed emergent surgery as soon as diagnosis was confirmed, because the patients required an IABP in order to control the progress of heart failure.

\section{Procedure}

Following median sternotomy and pericardiotomy, direct epicardial echocardiography was performed for verifying the position of the VSP. We established CPB with ascending aortic perfusion and right atrium drainage. A left ventricular venting tube was inserted through the right upper pulmonary vein. Under conditions of beating heart with $\mathrm{CPB}$, a 7-8 cm longitudinal left ventriculotomy was made parallel to the $\mathrm{LAD}$ at the infarcted wall and careful observation was carried out for verifying the expanse of infarction and the margin of healthy myocardium. The aorta was then cross-clamped and the heart was arrested with a cold crystalloid cardioplegic solution.

The flat patch was created from a bovine pericardium (Fig. 1A, B) or a Teflon sheet (Fig. 1C), which was cut into an oval shape of approximately $5 \times 6 \mathrm{~cm}$ to match the infracted ventricular septum including the VSP. The conic patch was made from a $10 \times 15$ $\mathrm{cm}$ bovine pericardium, which was sewed into a cylindrical state of approximately $5 \mathrm{~cm}$ in diameter to conically line the infracted myocardium of the left ventricle later.

The flat patch was sutured onto the ventricular septum using 10 or more mattress sutures with 2-0 polypropylene. At the basal and posterior sides, half of these sutures went first through the flat patch, then through the margin of healthy myocardium, and finally through the conic patch (Fig. 1A-C). In the first case, more of these sutures at the apical and anterior sides went through the flat patch, the infarcted septum and the right ventricular free wall (Fig. 1A). In the other two cases, this process was carried out when closing the left ventriculotomies, in which the left ventricular free walls were sutured together (Fig. 1B, C). Half of the bottom fringe of each conic patch had already been sutured to the basal septum and the posterior wall, so that the other half was sutured to the anterior and lateral free wall with 6 or more mattress sutures with 2-0 polypropylene (Fig. 1A-C). In addition, all mattress sutures for the conic patch were reinforced with running 3-0 polypropylene sutures. The conic patch was then sewed up into a conic state using a continuous 4-0 polypropylene suture in order to exclude the infracted myocardium of the left ventricle (Fig. 1 A, B, Fig. 2). The left ventriculotomy was buttressed with bovine pericardium and closed using 5 or more horizontal mattress and "over-and-over" 2-0 polypropylene sutures with Teflon felt strips. In the last two cases, the apical and anterior sides of the flat patch were sutured while closing the ventriculotomies at the same time (Fig. 1B, C). In the third case, the conic patch was also sutured with a ventriculotomy to create its conic state (Fig. 1C).
We did not excise the infarct tissue or apply any surgical glue. After the CPB was terminated, intraoperative transesophageal echocardiography verified that there was no residual VSP shunt. The mean operating time was 342 minutes (range 324-368 minutes). The mean CPB time was 219 minutes (range 216-225 minutes). The mean aortic cross-clamp time was 140 minutes (range 111-162 minutes).

\section{Results}

After surgeries, all patients recovered from shock and the IABPs were removed by the third postoperative day. The first and second cases were extubated at 1 week and discharged at 2 months. Transthoracic echocardiography demonstrated slight VSP shunts before discharge, which disappeared within 3 months. Unfortunately, the patient in the third case suffered from hypoxic ischemic encephalopathy due to preoperative cardiac arrest and died of multiple organ failure 2 months after operation.

\section{Discussion}

Cooley and colleagues ${ }^{2)}$ reported the first surgical repair of VSP in 1957. Daggett and associates ${ }^{3)}$ established the conventional patch repair with infarctectomy for acute VSP around 1980. Since then, various surgical techniques to overcome this fatal complication have been introduced, including such techniques as infarct exclusion techniques, double patch techniques, sandwich repairs, application of surgical glue, and the right ventricular (RV) approach, among others. ${ }^{4)}$ The recent evolution of these techniques has led to expectations of improved treatment outcomes, but the surgical mortality of acute VSP remained high, 38.3\% in Japan during 2010. ${ }^{1)}$ It has been considered, that in order to repair VSPs more successfully, two or more such techniques should be integrated.

An infarct exclusion technique with a single patch was proposed by Komeda, et al. in 1990.5) This technique consists of left ventriculotomy in the infarcted free wall, suturing a three-dimensional patch to the healthy myocardium without infarctectomy, and excluding the VSP and the infarcted myocardium from the left ventricle. This innovative technique has reduced the mortality and morbidity of acute VSP. David, et al. ${ }^{6}$ reported 6 operative deaths (13.6\%) and only one residual VSP in 44 patients. There is, however, the technical difficulty of distinguishing between healthy endocardium and infarction. The patches must be sutured onto the healthy endocardium to exclude the infarcted myocardium of the left ventricle. For that reason, the endocardium must be carefully observed under conditions of beating heart with $\mathrm{CPB}$. Our infarct exclusion procedure was then carried out under the condition of cardiac arrest. Another difficulty exists in tailoring a flat pericardial patch into the shape of the left ventricular cavity. Most surgeons require extended oper- 

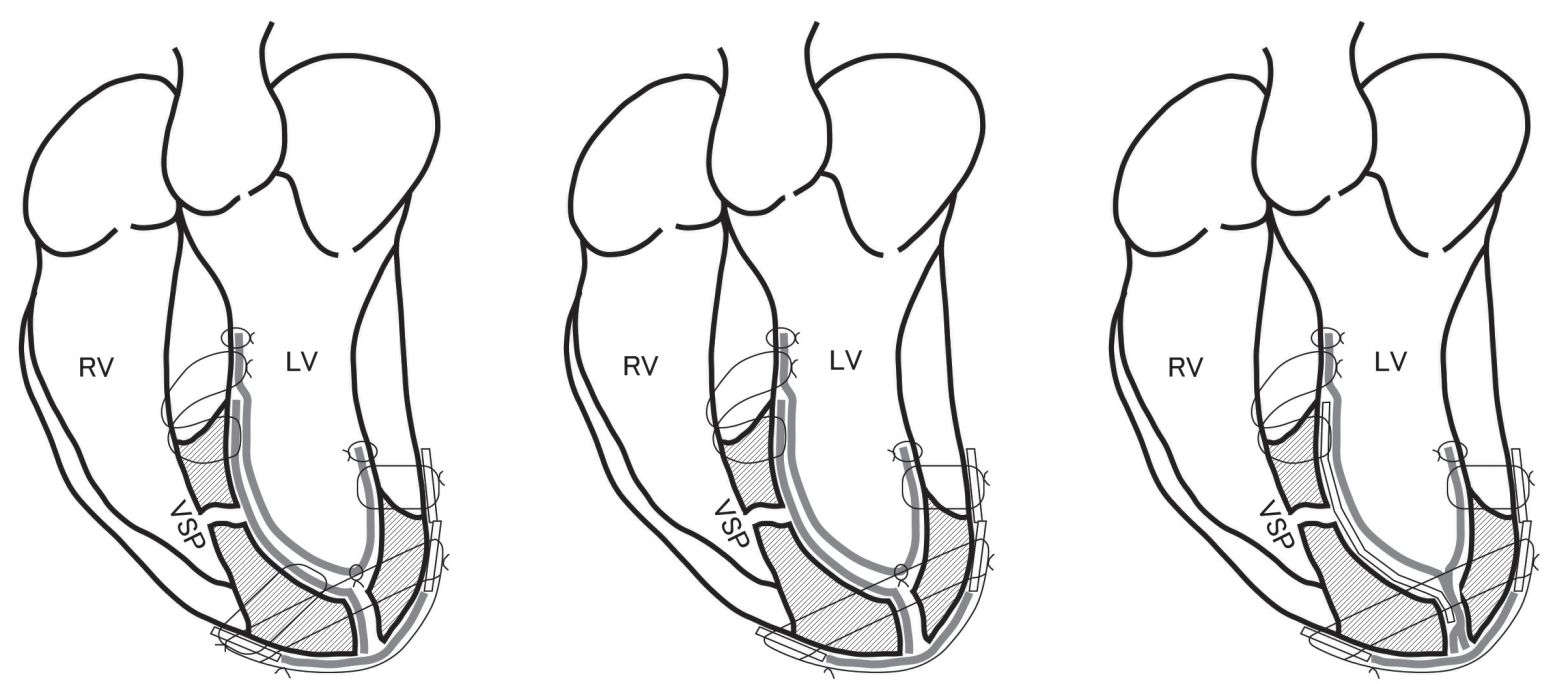

Fig. 1 VSP repair with flat and conic patches.

A: In the first case, we performed each process one after the other: suturing the apical half of the flat patch to the ventricular septum, sewing up the conic patch from a cylindrical state into a conic state, and closing the left ventriculotomy.

$\mathrm{B}$ : In the second case, the first and third processes were carried out simultaneously.

$\mathrm{C}$ : In the third case, the three processes were combined into one step.

VSP, ventricular septal perforation; RV, right ventricle; $L V$, left ventricle

, bovine pericardium; $\square$, Teflon

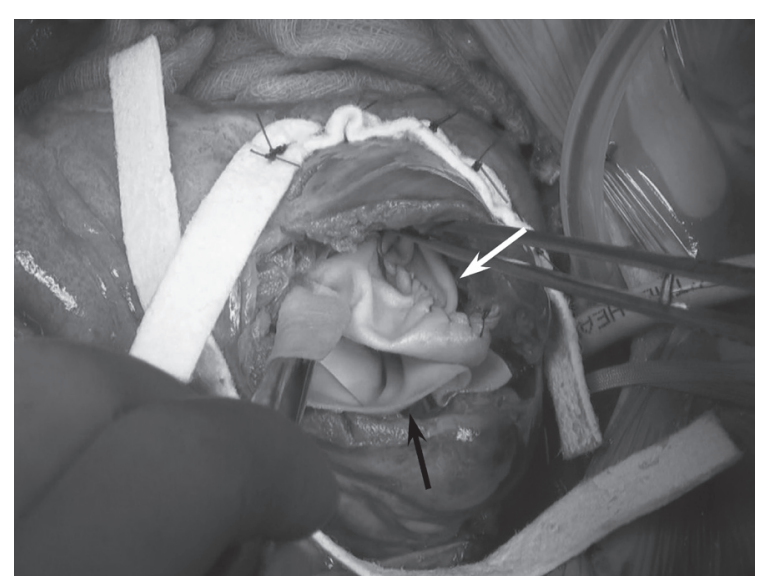

Fig. 2 Intraoperative picture just before closing the left ventriculotomy in the second case.

The conic patch (white arrow) looks like an apical state next to the flat patch (black arrow).

ating times with this original procedure and residual VSP shunts occur frequently. ${ }^{7}$ Many surgeons have modified the method of creating and suturing the three-dimensional patch on the inside of the left ventricle. Matsuda, et al. ${ }^{8}$ designed a single pericardial patch trimmed into a conic sack, and Shibata, et al. ${ }^{9}{ }^{9}$ devised two pericardial patches joined to make a single pouch.

On the other hand, in 1969 Iben, et al. ${ }^{10)}$ reported the double Teflon patch technique through a right vetriculotomy. Various double patch techniques and sandwich repairs using two patches have been introduced over the following decades.4, 7) Double block of VSP shunts might be another innovative technique for reducing residual shunts. Hisatomi, et al. ${ }^{7)}$ reported no operative death nor residual VSP in 9 patients treated with their double patch technique. Many surgeons have applied surgical glues such as gelatin-resorcin-formol (GRF) biological glue (Pharmacie Centrale, CHU, Henri Mondor, Creteil, France) to reinforce infarcted myocardium and to seal the space between double patches. $\left.{ }^{4}, 7\right)$ However, this conventional technique is not an infarct exclusion. There are concerns about bleeding from the left ventriculotomy, and future growth of a left ventricular aneurysm. During the 2000s, some surgeons combined an infarct exclusion technique and a double patch technique, ${ }^{11-13)}$ and we have done the same. Sugimoto, et al. ${ }^{13)}$ have reported such a technique using three pericardial patches, which was performed successfully for not only anterior but also posterior VSP. The combination of both the techniques is thought to be an ideal strategy for acute VSP, although further experience is needed to verify its efficacy.

If the hemodynamic conditions of a VSP patient allow, we wait for the chronic stage of AMI and repair VSP by suturing the firm tissue. However, most patients had suffered from cardiogenic shock in the emergency room and required an IABP before surgery. Over the past 10 years, ten of fifteen VSP surgeries were performed within 1 week of the onset of AMI in our hospital. Only two patients underwent surgery at the chronic stage, more than 1 month after the onset. Formerly, we performed the infarct exclusion technique with a single patch for acute VSP. This resulted in much residual shunt and high mortality $(67 \%)$. Therefore a double patch technique with flat patches was adopted for the cases that followed, but VSP recurred and heart failure dete- 
riorated. We thus recognized that considerable modification would be needed to improve treatment outcomes.

For the cases reported here we adopted flat and conic patches for infarct exclusion and double block of VSP shunts. The flat patches are for directly covering the infracted ventricular septum including the VSP. The conic patches are for three-dimensionally lining the infracted endocardium of the left ventricle. The former were made from bovine pericardium in the first 2 cases and a Teflon sheet in the third case. Teflon appears to be more suitable than pericardium for firmly supporting the infarcted ventricular septum. The latter were made from large bovine pericardium and sewed into a cylindrical state first, and then later sewed up into a conic state. Sugimoto, et al. ${ }^{13)}$ had devised two pericardial patches to create such a three-dimensional pouch. In the first case (Fig. 1A), each process was performed one after the other: suturing the apical half of the flat patch to the ventricular septum, sewing up the conic patch from the cylindrical state into a conic state, and closing the left ventriculotomy. In the second case (Fig. 1B), the first and third processes were carried out simultaneously in order to save time. In the third case (Fig. 1C), the three processes were combined into one step. Our procedure is still developing and should become one of the ideal options for acute VSP.

All our patients recovered from shock and retrieved enough cardiac function to revive, although slight VSP shunts recurred during the week after operation. Even if there is a residual shunt, the most important thing is to reduce shunt flow to the minimal range. Therefore, a conic patch was sutured onto the endocardium twice, using mattress sutures with 2-0 polypropylene and additional running 3-0 polypropylene sutures. Many surgeons have adopted surgical glue to reduce residual VSP shunts..$^{4,7,11-13)}$ We have wondered whether surgical glue would cause perioperative embolism such as cerebral infarction. GRF is the most popular glue, but it contains formaldehyde harmful to tissue. BioGlue (Cryolife Inc., Kennesaw, Georgia, USA) is a new surgical glue without toxic ingredients, but it hardens too quickly. Further improvement will be needed for us to decide on the application of surgical glue.

Some surgeons have reported successful VSP repairs using a sandwich technique and surgical glue via an RV approach. ${ }^{4)}$ This kind of procedure may offer another promising strategy for acute VSP. However, the RV approach is not so common because of the fear of postoperative RV failure. An RV approach was not chosen in the cases reported here either. The viable myocardium of the RV free wall should be preserved as much as possible. Further investigation is needed to verify the merits and demerits of this method.

\section{Conclusion}

We adopted flat and conic patches in performing VSP surgeries and have achieved acceptable outcomes. For this surgery, two important innovations have been made: an infarct exclusion technique and a double patch technique. The combination of both these techniques would to be an ideal strategy and our procedure presents one such option.

\section{Acknowledgements}

The authors wish to thank Avi Landau for language assistance.

\section{References}

1) Kuwano H, Amano J, Yokomise H: Thoracic and cardiovascular surgery in Japan during 2010: annual report by the Japanese association for thoracic surgery. Gen Thorac Cardiovasc Surg 2012; 60: 680-708

2) Cooley DA, Belmonte BA, Zeis LB, et al: Surgical repair of ruptured interventricular septum following acute myocardial infarction. Surgery 1957; 41: 930-937

3) Daggett WM, Buckley MJ, Akins CW, et al: Improved results of surgical management of postinfarction ventricular septal rupture. Ann Surg 1982; 196: 269-277

4) Isoda $S$, Osako M, Kimura T, et al: Surgical repair of postinfarction ventricular septal defects - 2013 update. Ann Thorac Cardiovasc Surg 2013; 19: 95-102

5) Komeda M, Fremes SE, David TE: Surgical repair of postinfarction ventricular septal defect. Circulation 1990; 82(Suppl IV): 243-247

6) David TE, Dale L, Sun Z: Postinfarction ventricular septal rupture: repair by endocardial patch with infarct exclusion. J Thorac Cardiovasc Surg 1995; 110: 1315-1322

7) Hisatomi K, Eishi K, Hamawaki M, et al: Double patch technique for closing acute ventricular septal perforation. Gen Thorac Cardiovasc Surg 2010; 58: 555-560

8) Matsuda K, Oda T, Terai H, et al: New surgical technique for repair of ventricular septal perforation. Ann Thorac Surg 1995; 60: $1430-1431$

9) Shibata T, Suehiro S, Ishikawa T, et al: Repair of postinfarction ventricular septal defect with joined endocardial patches. Ann Thorac Surg 1997; 63: 1165-1167

10) Iben AB, Pupello DF, Stinson EB, et al: Surgical treatment of postinfarction ventricular septal defects. Ann Thorac Surg 1969; 8: 252-262

11) Tabuchi N, Mizuno T, Kuriu K, et al: Double patch technique for repairing postinfarction ventricular septal defect. Jpn J Thorac Cardiovasc Surg 2001; 49: 264-266

12) Tanaka H, Hasegawa $S$, Sakamoto T, et al: Postinfarction ventricular septal perforation repair with endoventricular circular patch plasty using double patches and gelatin-resorcinol-formaldehyde biological glue. Eur J Cardiothorac Surg 2001; 19: 945-948

13) Sugimoto T, Yoshida S, Yamamoto K, et al: A modified infarct exclusion repair of posterior postinfarction ventricular perforation: triple-patch technique for postinfarction ventricular septal perforation in 2 female patients. Ann Thorac Cardiovasc Surg 2011; 17: 90-93 